\title{
Implementations and pilot comparison of primary low intensity shock calibration
}

\author{
Qiao Sun, Hong-bo Hu \\ National Institute of Metrology, Beisanhuandonglu 18, 100029 Beijing, China
}

\begin{abstract}
This paper first presents two main technical concerns for a possible low intensity shock comparison: variety of primary shock calibration systems and feasibility of comparison artifact. For the primary calibration system, the mechanical excitation includes hammer-anvil collision, pneumatic driven projectile impact and the Hopkinson bar. The shock pulses generated have a smooth monopole shape by the first two types with air bearings, but a dipole shape by the third type. For the comparison artifact, the standard accelerometer of a back-to-back type with a charge amplifier consists of an accelerometer measuring chain whose nonlinearity of amplitude and phase frequency responses is investigated. Based on the nonlinear fact of comparison artifact in the frequency domain and the spectrum range difference of mechanical excitations of the calibration system, strict comparison conditions had to be laid down for the measurement of shock sensitivity at specific acceleration levels and pulse durations.

The pilot comparison, coded as APMP.AUV.V-P1, is successfully organized by the Asia Pacific Metrology Programme. Some comparison results of both monopole excitation and dipole excitation are shown with the expanded uncertainty. The completion of this pilot comparison can serve as part of the basis for a planned key comparison targeted at a low intensity shock range at Consultative Committee level.
\end{abstract}

\section{Section: RESEARCH PAPER}

Keywords: Metrology; comparison; primary shock calibration; low intensity shock acceleration

Citation: Qiao sun, Hong-bo Hu, Implementations and pilot comparison of primary low intensity shock calibration, Acta IMEKO, vol. 5, no. 3, article 11, November 2016, identifier: IMEKO-ACTA-05 (2016)-03-11

Section Editor: Konrad Jedrzejewski, Warsaw University of Technology, Poland

Received January 13, 2016; In final form June 16, 2016; Published November 2016

Copyright: (C) 2016 IMEKO. This is an open-access article distributed under the terms of the Creative Commons Attribution 3.0 License, which permits unrestricted use, distribution, and reproduction in any medium, provided the original author and source are credited

Funding: This work was supported by General Administration of Quality Supervision, Inspection and Quarantine, the People's Republic of China.

Corresponding author: Qiao sun, e-mail: sunq@nim.ac.cn

\section{INTRODUCTION}

Accurate low intensity shock acceleration calibration is of special importance for automobile and civil industries worldwide. The Physikalisch-Technisches Bundesanstalt (PTB) set the first example of a successful implementation of low intensity shock calibration by laser interferometry [1]. Recent years have witnessed the establishment of a primary low intensity shock calibration standard and relevant scientific research projects at different metrology institutes of the APMP (Asia Pacific Metrology Programme), such as the National Metrology Institute of Japan (NMIJ) [2], the Industrial Technology Research Institute (ITRI) of Taiwan [3], the National Institute of Metrology of Thailand (NIMT), and the National Institute of Metrology (NIM), China [4].
The International Organization for Standardization (ISO) 16063-13[5] describes the primary low intensity shock calibration method, algorithm, and technical requirements, with an implementation example of a rigid body collision with excitations from $100 \mathrm{~m} / \mathrm{s}^{2}$ to $5000 \mathrm{~m} / \mathrm{s}^{2}$ and a pulse duration less than $10 \mathrm{~ms}$. However, in the metrological field of shocks, there was no formal comparison either at a Consultative Committee (CC) level or a Regional Metrology Organization Technical Committee (RMO TC) level. Therefore, the unification of the shock acceleration quantity was short of direct supporting evidence. The Consultative Committee of Acoustics, Ultrasound and Vibration (CCAUV) has already planned to conduct key comparisons for shock in its strategic planning programme for 2013 to 2023 [6], possibly one key comparison for low intensity and the other for high intensity. 
During the meeting of APMP TCAUV in 2011, the decision was taken to make preparations for a pilot comparison targeted at low intensity shock acceleration. Despite of scientific investigations of shock standards by individual institutes of the APMP, guest scientist research of the TC Initiative project as intra-APMP collaboration on this topic and dedicated sessions of TCAUV workshops in Japan in 2011 and in Thailand in 2014 made a direct contribution to the successful completion of this APMP TCAUV pilot comparison of low intensity shock, coded as APMP.AUV.V-P1 with participants from NIM, ITRI, NIMT and SPEKTRA (German DKD Lab) [7].

In this paper, the implementations of three different types of shock excitations suitable for laser interferometry are described. Rationale of strict comparison conditions are explained in detail. The comparison results are presented in two groups: shock sensitivities of monopole excitation from $500 \mathrm{~m} / \mathrm{s}^{2}$ to $5000 \mathrm{~m} / \mathrm{s}^{2}$ and shock sensitivities of dipole excitation at 1000 $\mathrm{m} / \mathrm{s}^{2}$. The organization of this pilot comparison by the APMP TCAUV has proved, among other things, the credibility of the primary low intensity shock calibration capability of the participants.

\section{IMPLEMENTATIONS OF A LOW INTENSITY SHOCK CALIBRATION SYSTEM}

The primary low intensity shock calibration system consists of three main parts: shock excitation, laser interferometer and signal acquisition and data processing. For the laser interferometer, either homodyne or heterodyne can meet the requirement as primary measurement standard of acceleration for the calibration system. The technology is available in previous research, such as in [1]-[5]. Signal acquisition and data processing algorithms and procedures are also covered in detail in [1]-[5]. The different shock excitation devices for primary laser interferometry calibration are of particular interest for the investigation of the pilot comparison in that their frequency spectra can be quite different. Therefore, three different types of shock excitation devices which are employed in the pilot comparison are described.

\subsection{Hammer-anvil excitation device}

The shock excitation device based on a hammer-anvil mechanical collision is called shock machine and its basic working principle is well explained in [5]. The actual implementation is verified. PTB uses a spring unit as exciter to provide the original shock force in [1]. NMIJ uses an air pressure exciter [2] and ITRI uses an electromagnetic exciter [3]. The NIM's shock excitation device consists of an electromagnetic exciter and a pneumatic exciter. This combination as a mechanical power supply of the excitation device can deliver a wide range of shock acceleration levels and wider pulse durations [4]. All these four versions of implementation are placed in a horizontal position. It is worth noting that a high resonant frequency airborne hammer and anvil as moving parts of the excitation device is a precondition to generate a high-quality monopole shock pulse. The thin stiff air films can largely reduce mechanical disturbances from other parts of the excitation device, and avoid asymmetric impact forces to the anvil. As a result, the anvil can move rectilinearly with less rotational motion and produce repeatable shock pulses for calibration. Figure 1 gives an instance of the primary shock calibration system based on the hammer-anvil excitation device from NIM.

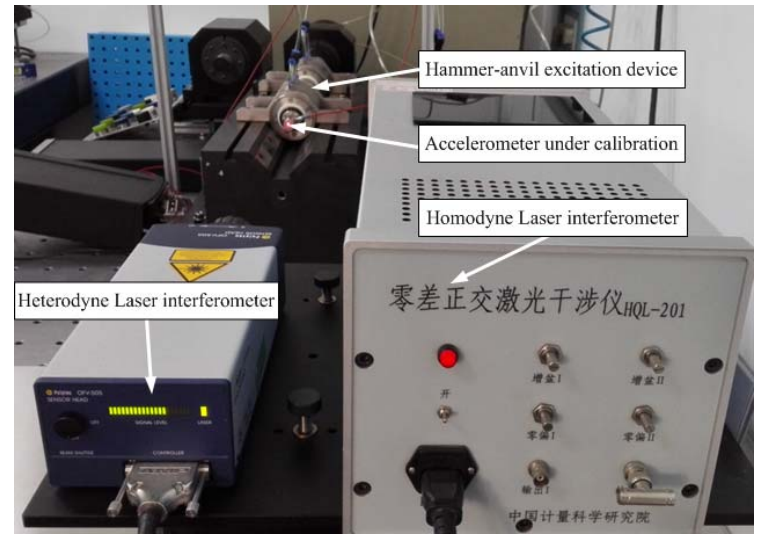

Figure 1. Photo of NIM's primary shock calibration system based on a hammer-anvil excitation device.

The structure of the shock excitation device is shown in Figure 2. In this figure, the shock exciter is the original part to supply mechanical power in the shock excitation device. The amount of output power is the decisive factor to determine the acceleration level and pulse duration of the shock pulse generated. Besides, good controllability of the power supply contributes to good repeatability of the acceleration level generated. Two shock exciters are deployed. The electromagnetic exciter is under precise control to produce an expected amount of power. The maximum output force is 9800 $\mathrm{N}$. Responding to a voltage input set by the controller, the electromagnetic exciter can drive the hammer at a speed from $0.08 \mathrm{~m} / \mathrm{s}$ to $4.06 \mathrm{~m} / \mathrm{s}$, whose repeatability is better than $1 \%$. However, limited by its maximum power, the electromagnetic exciter is only used to produce an acceleration level below 5000 $\mathrm{m} / \mathrm{s}^{2}$. The pneumatic exciter can produce a power supply corresponding to an air pressure range from 0.49 bar to 4.9 bar. By adjusting the air pressure inside the air tank and regulating the opening time of the electric valve, the pneumatic exciter can drive the hammer at the high speed limit of $5 \mathrm{~m} / \mathrm{s}$, which results in the highest generation of shock acceleration level above $10000 \mathrm{~m} / \mathrm{s}^{2}$.

Airborne hammer and anvil are moving parts of the shock machine. They are made of titanium and aluminium alloy respectively, with $30 \mathrm{~mm}$ in diameter and $200 \mathrm{~mm}$ in length. Each titanium hammer and anvil weighs $1.27 \mathrm{~kg}$. Their longitudinal resonant frequency of the first order is about 12 $\mathrm{kHz}$ obtained by special tests. This resonant frequency limits the minimum shock pulse duration to about $0.5 \mathrm{~ms}$ generated by the shock machine, with its most energy components in the low frequency range. Since the collision caused by their free

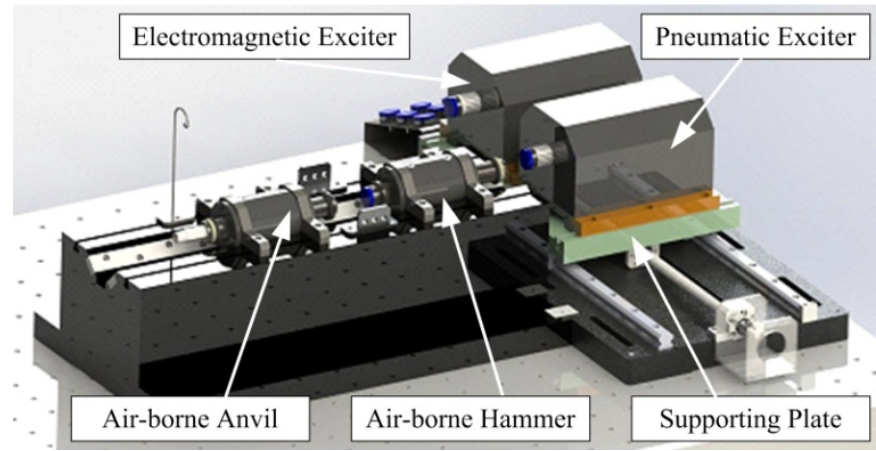

Figure 2. Model of the shock excitation device. 
motion and direct impact is a precondition for a high-quality shock pulse, both the hammer and the anvil are equipped with air bearings in such a way that their centerlines are aligned within a tolerance of $\pm 0.2 \mathrm{~mm}$. Air bearings of $30 \mathrm{~mm}$ inner diameter from New Way Co. are used. Stiff air films less than $10 \mu \mathrm{m}$ can significantly reduce negative influences from resonances of other parts of the shock machine structure, and avoid asymmetric impact forces to the anvil to a large extent. Therefore, the anvil can move rectilinearly without rotational motion, and produce repeatable shock pulses for calibration purposes.

The shock machine is based on solid body collision of hammer and anvil to produce excitation of shock pulses. But if the airborne hammer and anvil collides directly, it will cause strong resonant motions, and therefore the accelerometer mounted at the end surface of the anvil will provide unreliable output signals which cause inaccurate measurement results. This adverse influence must be avoided for accurate calibration results by attaching a cushioning pad to the hammer. The cushioning pad, likewise a damping isolator to absorb highfrequency noisy motions, acts as a shock pulse generator to produce desired pulse shapes with an expected acceleration level and pulse duration. This object is achieved by applying three sets of cushioning pads of different materials.

It is worth noting that different cushion pads of different materials or different hardnesses or thicknesses can generate shock pulses of different shapes, acceleration levels or pulse durations, due to their own damping properties. Therefore, each cushioning pad functions well to produce shock pulses at certain acceleration levels. Beyond this working range, the pulse shape generated may be distorted and thus cause poor calibration results.

\subsection{Pneumatic projectile excitation device}

The pneumatically driven projectile excitation device is a vertical implementation for good-quality monopole shock pulses in the pilot comparison. A projectile accelerated by pressurized air functions as hammer. While the air pressure remains constant, the kinetic energy of the projectile can be controlled by a motor driven mechanical stop that allows a precise adjustment of the projectiles starting position and thus of the distance over which it is accelerated. Therefore, the repeatability of the shock pulse generated by this excitation device is good under the full automatic control of the target acceleration level. To be used as mechanical excitation source for laser interferometry measurements, an air bearing is equipped with the mounting part of the accelerometer to reduce transverse motions caused by the impact of the projectile. Figure 3 shows the primary shock calibration system based on a pneumatic projectile excitation device from SPEKTRA.

\subsection{Hopkinson bar excitation device}

The Hopkinson bar excitation device is based on wave propagation and reflection characteristics inside a long thin bar and can generate dipole shock pulses normally in the acceleration range from $1000 \mathrm{~m} / \mathrm{s}^{2}$ to $100000 \mathrm{~m} / \mathrm{s}^{2}$ as described in [5]. By careful determination of the dimension and length of the titanium Hopkinson bar and application of a piezoelectric actuator as exciter, a shock acceleration range from $200 \mathrm{~m} / \mathrm{s}^{2}$ to $40000 \mathrm{~m} / \mathrm{s}^{2}$ can be achieved. However, the pulse duration of the half sine shape is narrower than $0.2 \mathrm{~ms}$ [8].

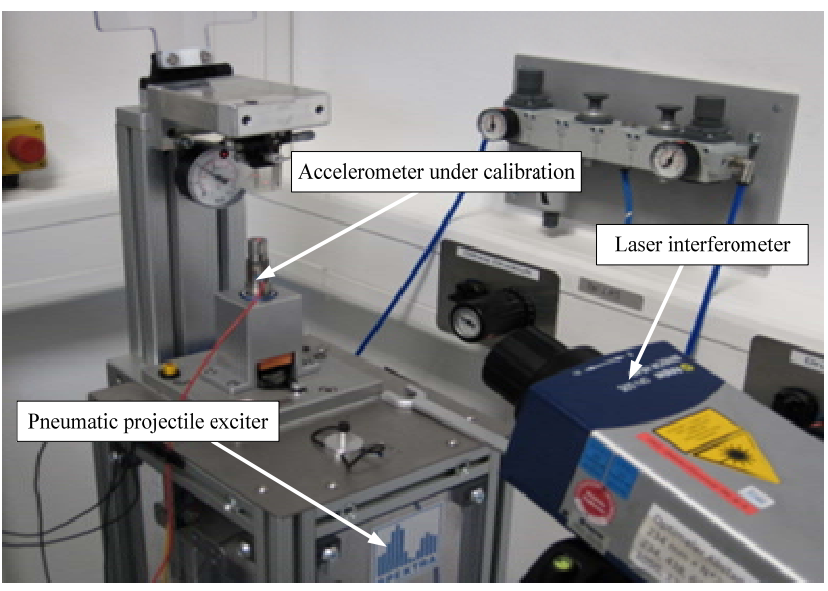

Figure 3. Photo of the SPEKTRA's primary shock calibration system based on a pneumatic projectile excitation device.

The main parts of the Hopkinson bar excitation device include a Hopkinson Bar, a piezoelectric actuator and a reaction mass. When a driving voltage is applied to the actuator, the piezo-stack changes its length and a reaction force is generated by the reaction mass according to Newton's 2nd law. This force acts as the input force on the end surface of the Hopkinson Bar. The acceleration generated at the other end of the bar can be quite accurate because the driving voltage can be precisely controlled. Figure 4 shows the primary shock calibration system based on the pneumatic projectile excitation device from NIMT.

\section{PILOT COMPARISON}

\subsection{Background of the comparison}

The accurate measurement of low intensity shock acceleration is vital in certain applications, for example car crash tests. Efforts to decrease the losses in human lives on the roads during the $1950 \mathrm{~s}$ led to an increased research into the biomechanics of the head impact. A break-through was made with the introduction of the Wayne State Tolerance Curve [9].

This curve was interpreted and a weighted injury criterion was developed. This criterion was later transformed into the Head Injury Criterion (HIC) to improve the crashworthiness of cars. HIC is a measure of the likelihood of head injury arising from an impact. The HIC can be used to assess safety related to vehicles, and it is defined as:

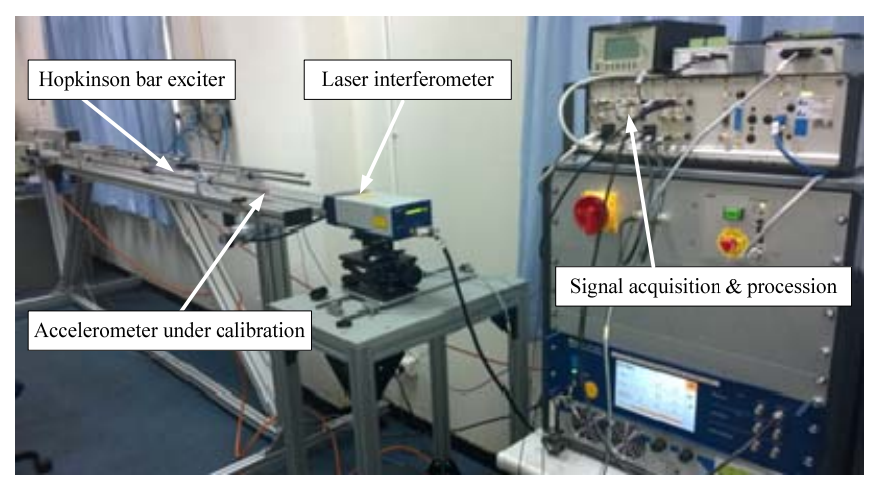

Figure 4. Photo of the NIMT's primary shock calibration system based on a Hopkinson bar excitation device. 


$$
H I C=\left\{\left[\frac{1}{t_{2}-t_{1}} \int_{t_{1}}^{t_{2}} a(t) d t\right]^{2.5}\left(t_{2}-t_{1}\right)\right\}_{\max },
$$

where $t_{1}$ and $t_{2}$ are any two arbitrary times during the acceleration pulse. In 1986, the time interval over which HIC is calculated was limited to $36 \mathrm{~ms}$. Shock acceleration is measured in standard gravity acceleration, shown in Figure 5.

Normally the variable is derived from the acceleration/time history of an accelerometer mounted at the centre of gravity of a dummy's head, like in the car crash test photo of Figure 5, when the dummy is exposed to crash forces. This means that the HIC includes the effects of head acceleration and the duration of the acceleration. Large accelerations of up to 5000 $\mathrm{m} / \mathrm{s}^{2}$ may be tolerated for very short durations of less than 1 ms.

Therefore, a low intensity shock comparison is well justified with the acceleration range from $500 \mathrm{~m} / \mathrm{s}^{2}$ to $5000 \mathrm{~m} / \mathrm{s}^{2}$ and a duration of monopole shock pulse from 0.3 to $3 \mathrm{~ms}$.

\subsection{Comparison feasibility}

It was found previously by PTB that different shock sensitivities of the same accelerometer under the same level of shock acceleration were obtained when it was exposed to the excitation of a hammer-anvil device and a Hopkinson bar device, respectively. The sensitivity difference was about $4 \%$ at about $5000 \mathrm{~m} / \mathrm{s}^{2}$. Figure 6 reveals the cause of this sensitivity difference. The excitation of rigid body motion, the impact from hammer-anvil or pneumatic projectile, falls into a narrow low frequency range of the spectrum while the excitation by the Hopkinson bar covers a wider spectrum range from low to high frequencies. The sensitivity magnitude frequency response of the accelerometer only has a narrow working range of constant sensitivity at low frequency. This effect plays an increasing role when the shock pulse duration decreases and therefore covers a wide frequency range, which finally results in obvious sensitivity difference by two different excitations.

Therefore, pulse shape, pulse duration and acceleration level should be restricted for the feasibility of the pilot low intensity
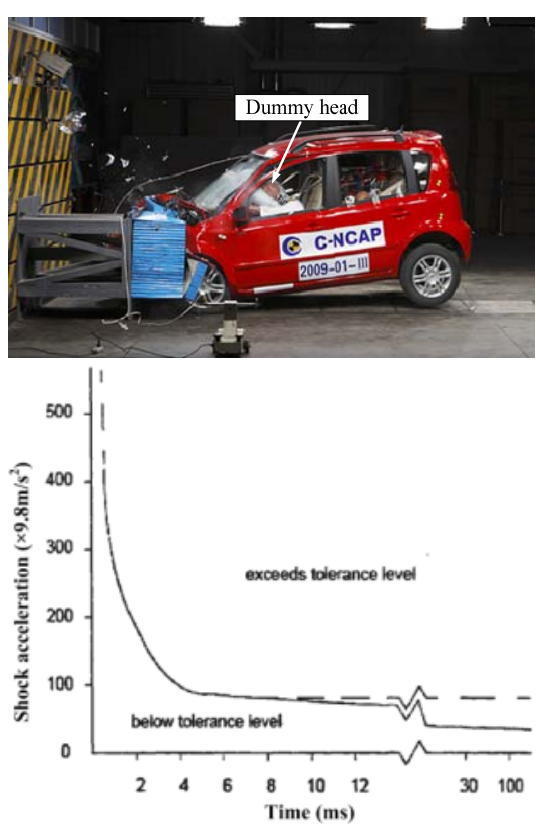

Figure 5. Wayne State Tolerance Curve and Photo of car crash test in China.

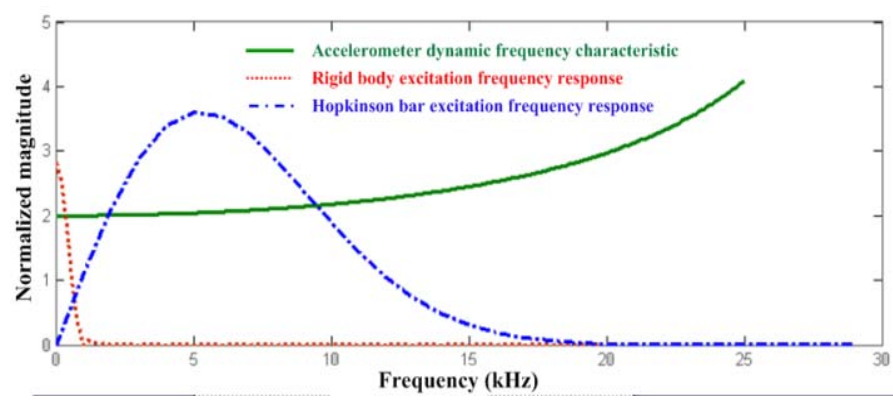

Figure 6. Frequency responses of the accelerometer, the rigid body and the Hopkinson bar.

shock comparison. Hammer-anvil excitation and pneumatic projectile excitation can generate monopole pulses and fall into the same group of rigid body motion. Hopkinson bar excitation should be treated into a different group. It should be noted that pulse duration is defined as time width between rising edge point and falling edge point at a $10 \%$ level of peak acceleration.

For the monopole group, participants are supposed to measure at the following acceleration levels (all values in $\mathrm{m} / \mathrm{s}^{2}$ ): $500,1000,2000,3000,4000,5000$. A series of $0.5 \mathrm{~ms}, 1 \mathrm{~ms}, 1.5$ $\mathrm{ms}$ and $2 \mathrm{~ms}$ of shock pulses are recommended, with a reference of $2 \mathrm{~ms}$ at an acceleration of $1000 \mathrm{~m} / \mathrm{s}^{2}$.

For the dipole group, participants are supposed to measure at $1000 \mathrm{~m} / \mathrm{s}^{2}$. A series of $0.03 \mathrm{~ms}, 0.05 \mathrm{~ms}, 0.07 \mathrm{~ms}, 0.10 \mathrm{~ms}$ (reference), $0.15 \mathrm{~ms}$ and $0.20 \mathrm{~ms}$ are recommended.

\subsection{Comparison results}

For the purpose of the comparison the pilot laboratory selected an ENDEVCO 2270 (SN: 10466) with a Brüel \& Kjær charge amplifier 2692 (SN: 2752215) as Accelerometer Chain of which monitoring data for 6 months were available and of which data were not included in any published international cooperation work. For this comparison, NIM used its hammeranvil calibration system and Hopkinson bar calibration system; ITRI used its hammer-anvil calibration system; NIMT used its Hopkinson bar calibration system; and SPEKTRA used its pneumatic projectile calibration system and Hopkinson bar calibration system. In Table 1 and 2, the calibration results under monopole and dipole excitations are presented.

The weighted mean was agreed upon by all participants to calculate the Pilot Comparison Reference Values (PCRV) for the APMP.AUV.V-P1 data. PCRVs were calculated separately at each acceleration or pulse duration point for the Accelerometer Chain. Four typical degrees of equivalence with respect to PCRVs of the participants are shown in Figure 7 and 8. The degrees of equivalence also support the uncertainty of the measurements of the participants at other acceleration levels and pulse durations.

\section{CONCLUSIONS}

Based on the investigation work on the primary low intensity shock calibration technique and feasibility of a pilot comparison, TCAUV of APMP has successfully conducted a pilot comparison of shock acceleration sensitivity at low intensity shock accelerations from $500 \mathrm{~m} / \mathrm{s}^{2}$ to $5000 \mathrm{~m} / \mathrm{s}^{2}$. Laser interferometry is a necessity as a measurement standard of the calibration system. Three different types of mechanical shock excitations are employed by the participants.

The comparison results are divided into two groups by the excitation pulse shape: the monopole and the dipole group. The 
Table 1. Calibration results of the participants for voltage sensitivities Sva under monopole shock excitation with expanded relative uncertainty $U c(k=2)$.

\begin{tabular}{|c|c|c|c|c|c|c|c|}
\hline \multirow{2}{*}{$\begin{array}{l}\text { Acceleration } \\
\qquad\left(\mathrm{m} / \mathrm{s}^{2}\right)\end{array}$} & \multirow{2}{*}{$\begin{array}{l}\text { Pulse duration } \\
\text { (ms) }\end{array}$} & \multicolumn{2}{|c|}{ NIM } & \multicolumn{2}{|c|}{ ITRI } & \multicolumn{2}{|c|}{ SPEKTRA } \\
\hline & & $\begin{array}{c}S_{v a} \\
\left(\mathrm{mV} /\left(\mathrm{m} / \mathrm{s}^{2}\right)\right)\end{array}$ & $U_{c}(\%)$ & $\begin{array}{c}S_{v a} \\
\left(\mathrm{mV} /\left(\mathrm{m} / \mathrm{s}^{2}\right)\right)\end{array}$ & $U_{c}(\%)$ & $\begin{array}{c}S_{v a} \\
\left(\mathrm{mV} /\left(\mathrm{m} / \mathrm{s}^{2}\right)\right)\end{array}$ & $U_{c}(\%)$ \\
\hline 500 & 3.0 & 0.1967 & 0.5 & 0.1966 & 1.0 & 0.19727 & 0.5 \\
\hline 1000 & 2.0 & 0.1968 & 0.5 & 0.1970 & 1.0 & 0.19746 & 0.5 \\
\hline 2000 & 1.5 & 0.1970 & 0.5 & 0.1971 & 1.0 & 0.19749 & 0.7 \\
\hline 3000 & 1.0 & 0.1973 & 0.5 & 0.1971 & 1.0 & 0.19755 & 0.7 \\
\hline 4000 & 1.0 & 0.1973 & 0.5 & 0.1971 & 1.0 & 0.19735 & 0.7 \\
\hline 5000 & 0.8 & 0.1973 & 0.5 & 0.1971 & 1.0 & 0.19734 & 0.7 \\
\hline
\end{tabular}

Table 2. Calibration results of the participants for voltage sensitivities Sva under dipole shock excitation with expanded relative uncertainty $U c(k=2)$.

\begin{tabular}{|c|c|c|c|c|c|c|c|}
\hline \multirow{2}{*}{$\begin{array}{c}\text { Acceleration } \\
\left(\mathrm{m} / \mathrm{s}^{2}\right)\end{array}$} & \multirow{2}{*}{$\begin{array}{l}\text { Pulse duration } \\
\text { (ms) }\end{array}$} & \multicolumn{2}{|c|}{ NIM } & \multicolumn{2}{|c|}{ NIMT } & \multicolumn{2}{|c|}{ SPEKTRA } \\
\hline & & $\begin{array}{c}S_{v a} \\
\left(\mathrm{mV} /\left(\mathrm{m} / \mathrm{s}^{2}\right)\right)\end{array}$ & $U_{c}(\%)$ & $\begin{array}{c}S_{v a} \\
\left(\mathrm{mV} /\left(\mathrm{m} / \mathrm{s}^{2}\right)\right)\end{array}$ & $U_{c}(\%)$ & $\begin{array}{c}S_{v a} \\
\left(\mathrm{mV} /\left(\mathrm{m} / \mathrm{s}^{2}\right)\right)\end{array}$ & $U_{c}(\%)$ \\
\hline 1000 & 0.20 & 0.1972 & 0.5 & 0.1976 & 0.6 & 0.19765 & 0.5 \\
\hline 1000 & 0.15 & 0.1973 & 0.5 & 0.1977 & 0.6 & 0.19744 & 0.5 \\
\hline 1000 & 0.10 & 0.1976 & 1.0 & 0.1976 & 0.7 & 0.19765 & 0.5 \\
\hline 1000 & 0.07 & 0.1981 & 1.0 & 0.1976 & 1.0 & 0.19776 & 0.8 \\
\hline 1000 & 0.05 & 0.1985 & 1.5 & 0.1977 & 1.0 & 0.19758 & 0.8 \\
\hline 1000 & 0.03 & 0.1990 & 1.5 & 0.1985 & 1.0 & 0.19718 & 0.8 \\
\hline
\end{tabular}
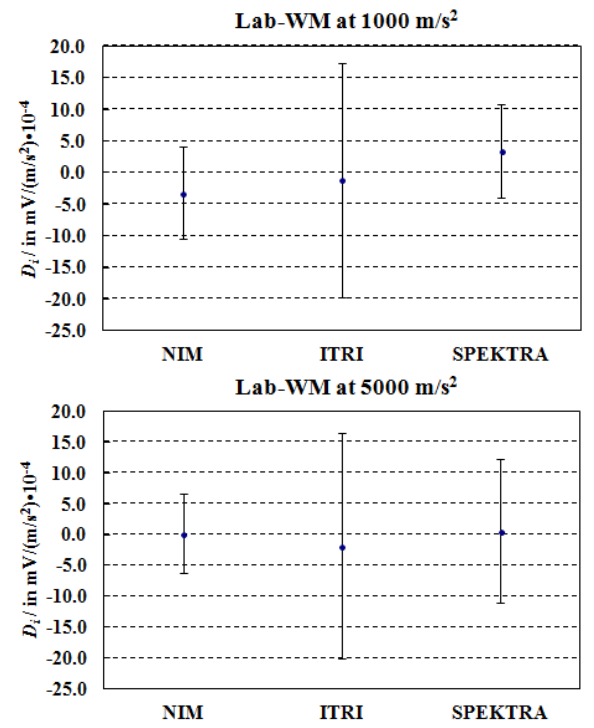

Figure 7. Degree of equivalence for voltage sensitivities under monopole shock excitation at $1000 \mathrm{~m} / \mathrm{s}^{2}, 2.0 \mathrm{~ms}$ and $5000 \mathrm{~m} / \mathrm{s}^{2}, 0.8 \mathrm{~ms}$.

reported sensitivities and associated uncertainties from four participants NIM, ITRI, NIMT and SPEKTRA are used for the calculation of mean values of the pilot comparison results and their associated uncertainties, as well as the deviations from the mean values with associated uncertainties. The degrees of equivalence calculated from the measurement results by the four participants support the measurement uncertainty reported by them at all acceleration levels and pulse durations specified in the technical protocol.

The successful completion of this pilot comparison can serve, among other things, as part of the basis for a planned key
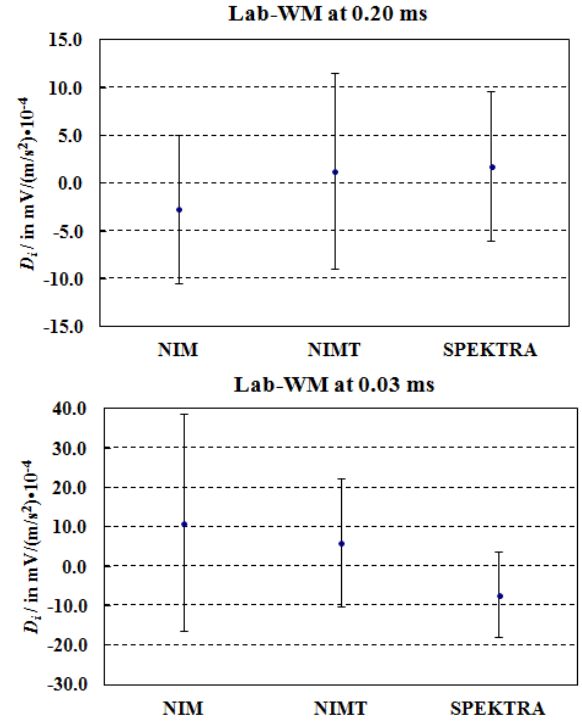

Figure 8. Degree of equivalence for voltage sensitivities under dipole shock excitation at $1000 \mathrm{~m} / \mathrm{s}^{2}, 0.20 \mathrm{~ms}$ and $0.03 \mathrm{~ms}$.

comparison coded as CCAUV.V-K4 targeted at a low intensity shock range at CCAUV.

\section{ACKNOWLEDGEMENT}

This work was supported in part by the General Administration of Quality Supervision, Inspection and Quarantine of the People's Republic of China under Contract No. ALC1201 and was partly funded by the APMP TC Initiative project No. TCI-2011-01-TCAUV and by the APMP DEC project 2014. The authors would like to give special 
thanks to colleagues in ITRI, NIMT, NMIJ and SPEKTRA for their support and cooperation in this comparison, related research work and workshop activities.

\section{REFERENCES}

[1] Link A, von Martens HJ, "Calibration of accelerometers by shock excitation and laser interferometry", Shock Vib, 2000 7:101-112.

[2] H Nozato, T Usuda, A Oota, T Ishigami, "Calibration of vibration pick-ups with laser interferometry: part IV Development of a shock acceleration exciter and calibration system", Measurement Sci. Technol. 2010 21:065107.

[3] Yu-Chung Huang, Jiun-Kai Chen, Hsin-Chia Ho, Chung-sheng $\mathrm{Tu}$, Chao-Jun Chen, "The set up of primary calibration system for shock acceleration in NML", Measurement, 2012 45: 23832387.
[4] Qiao Sun, Jian-lin Wang, Hong-bo Hu, “A primary standard for low-g shock calibration by laser interferometry", Measurement Sci. Technol. 2014 25:075003.

[5] ISO 16063-13, Methods for the calibration of vibration and shock transducers. Part 13: Primary shock calibration using laser interferometry, 2001.

[6] CIPM CCAUV, "Strategy document for rolling programme development for 2013 to 2023", www.bipm.org/en/committees/cc/ccauv.

[7] Qiao Sun, HongBo Hu. "Final Report on Pilot Comparison of Low Intensity Shock APMP.AUV.V-P1". Metrologia Tech. Suppl., 2015, 52: 09002.

[8] SPEKTRA, Data sheet of SE-220 HOP-MS Hopkinson Bar Shock Exciter, www.spektra-dresden.com/spektra.

[9] Lissner HR, Lebow M, Evans FG 1960 "Experimental studies on the relation between acceleration and intracranial pressure change" in men Surgery Gynecology and obstetrics 111: 329. 